\title{
Retraction Note to: Screening and differential physiological responses of tomato (Solanum lycopersicum L.) to drought stress
}

\author{
Rowland M. Kamanga ${ }^{1}$
}

Published online: 13 June 2021

(C) Indian Society for Plant Physiology 2021

Retraction to: Plant Physiology Reports (2020) 25(3):472-482

https://doi.org/10.1007/s40502-020-00532-6

The author has retracted this article. The author stated he did not have the sole ownership rights of the data in this article, and did not have the appropriate permission to publish it.

The original article can be found online at https:// doi.org/10.1007/s40502-020-00532-6.

Rowland M. Kamanga

rowlandkamanga5@gmail.com

1 Graduate School of Biosphere Science, Hiroshima

University, 1-4-4 Kagamiyama, Higashi-Hiroshima,

Hiroshima, Japan 